\title{
Extratos etanólicos da manga como antioxidantes para frangos de corte
}

\author{
Ednardo Rodrigues Freitas( ${ }^{(1)}$, Ângela da Silva Borges ${ }^{(2)}$, Maria Teresa Sales Trevisan ${ }^{(3)}$, \\ Pedro Henrique Watanabe ${ }^{(1)}$, André Luís da Cunha ${ }^{(2)}$, Ana Lúcia Fernandes Pereira ${ }^{(2)}$, \\ Virgínia Kelly Abreu(2) e Germano Augusto Jerônimo do Nascimento(1)
}

\begin{abstract}
(1)Universidade Federal do Ceará (UFC), Centro de Ciências Agrárias, Departamento de Zootecnia, Campus do Pici, Avenida Mister Hull, no 2.977, Caixa Postal 12 168, CEP 60021-970 Fortaleza, CE. E-mail: ednardo@ufc.br, pedrowatanabe@ufc.br, germanoaugustoj@ufc.br (2)Universidade Federal do Maranhão, Departamento de Engenharia de Alimentos, Campus Universitário de Imperatriz, Rua Urbano Santos, s/no, CEP 65900-410 Imperatriz, MA. E-mail: angelasborges@yahoo.com.br, andreluiscunha@yahoo.com.br, anafernandesp@yahoo.com.br, vkelly_abreu@yahoo.com.br (3)UFC, Departamento de Química Orgânica e Inorgânica. E-mail: trevisan@ufc.br
\end{abstract}

Resumo - O objetivo deste trabalho foi avaliar o efeito da inclusão de extratos etanólicos na ração, obtidos do caroço e da casca da manga, sobre o desempenho de frangos e a oxidação lipídica da carne. Foram utilizados 360 pintos machos da linhagem Ross 308, de um dia de idade, distribuídos em delineamento inteiramente casualizado, com seis tratamentos e seis repetições de dez aves. Os tratamentos consistiram de: ração sem adição de antioxidante (controle); ração com adição de 200 ppm do antioxidante butilato de hidroxitolueno (BHT); ração com 200 ou 400 ppm de extrato da casca da manga (ECAS); e ração com 200 ou 400 ppm de extrato do caroço da manga (ECAR). A adição de BHT ou dos extratos da manga não influenciou significativamente o consumo de ração, o ganho de peso e a conversão alimentar. A adição de BHT e a de 400 ppm de ECAR proporcionaram maior estabilidade lipídica da carne fresca, mensurada pelas substâncias reativas ao ácido tiobarbitúrico antes do armazenamento. O extrato etanólico do caroço da manga, na dosagem de 200 e 400 ppm, retarda a oxidação lipídica da carne de frangos armazenada por 15 dias.

Termos para indexação: Gallus gallus, Mangifera indica, antioxidantes naturais, mangiferina, oxidação lipídica, qualidade da carne.

\section{Ethanol extracts of mango as antioxidants for broiler chicken}

\begin{abstract}
The objective of this work was to evaluate the effect of the diet inclusion of ethanol extracts, obtained from mango seed and peel, on the performance of broilers and on the lipid oxidation of meat. Three hundred and sisty one-day-old male chicks of the strain Ross 308 were distributed in a completely randomized design, with six treatments and six replicates of ten birds. Treatments consisted of: diet without antioxidant (control); diet with addition of $200 \mathrm{ppm}$ of the antioxidant butylhydroxytoluene (BHT); diet with 200 or $400 \mathrm{ppm}$ extract of mango peel (ECAS); and diet with 200 or 400 ppm extract of mango seed (ECAR). The addition of BHT or mango extracts did not significantly affect feed intake, weight gain, and feed conversion. The addition of BHT and of $400 \mathrm{ppm}$ of ECAR provided low lipid oxidation in fresh meat, measured by thiobarbituric acid reactive substances before storage. Ethanol extract of mango seed, at 200 and $400 \mathrm{ppm}$ dosage, delays lipid oxidation of chicken meat stored for 15 days.
\end{abstract}

Index terms: Gallus gallus, Mangifera indica, natural antioxidants, mangiferin, lipid oxidation, meat quality.

\section{Introdução}

A oxidação lipídica é a deterioração de componentes importantes dos alimentos, como ácidos graxos essenciais e vitaminas lipossolúveis, que afetam sua qualidade, aroma, sabor e valor nutricional, além de produzir substâncias potencialmente tóxicas, como malonaldeídos e óxidos de colesterol (Gray et al., 1996). Em rações para animais com ingredientes que apresentam elevada quantidade de ácidos graxos insaturados, como óleos de origem vegetal, a utilização de antioxidantes sintéticos ou naturais visa proteger seus constituintes dos efeitos da oxidação lipídica (Mariutti \& Bragagnolo, 2009).

Assim, a prevenção dos danos oxidativos que possam ocorrer durante o armazenamento das rações até o momento do consumo pelas aves, com a adição de antioxidantes aos ingredientes ou às rações, permite a manutenção dos valores nutricionais e energéticos das dietas (Fischer et al., 2005). 
É possível estender os benefícios do uso de antioxidantes na alimentação dos frangos para proteger a carne, já que esta, em razão do seu conteúdo em ácidos graxos poli-insaturados, é particularmente suscetível à deterioração oxidativa, um dos principais fatores limitantes da qualidade, da aceitabilidade e da estabilidade comercial da carne e dos produtos cárneos avícolas (Bou et al., 2001).

Os antioxidantes sintéticos comumente usados nas rações são o butilato de hidroxianisol (BHA) e o butilato de hidroxitolueno (BHT). Entretanto, estudos têm mostrado que os antioxidantes sintéticos, em doses altas, podem causar alterações hepáticas, elevada proliferação do retículo endoplasmático (Durán \& Padilha, 1993) e ter potencial efeito carcinogênico (Chen et al., 1992). Portanto, tem-se aumentado o número de pesquisas com compostos antioxidantes naturais de diferentes partes das plantas, principalmente dos que apresentam, em sua composição, componentes fenólicos, como flavonoides, ácidos fenólicos e tocoferol, que retardam a oxidação e apresentam sinergismo com antioxidantes sintéticos (Melo et al., 2003). Porém, é importante que estes aditivos não tenham efeito adverso no desempenho dos animais e na qualidade da carne.

A manga (Mangifera indica L.) é uma fruta tropical que apresenta, na casca e no caroço, componentes que atuam como antioxidantes naturais, com destaque para a provitamina $\mathrm{A}$, na forma de $\beta$-caroteno; vitaminas C e E (Puravankara et al., 2000; Abdalla et al., 2007; Ajila et al., 2007); e o composto fenol glicosilxantona, sob a forma de mangiferina, de atividade antioxidante (Puravankara et al., 2000; Bernardini et al., 2005; Abdalla et al., 2007). Barreto et al. (2008), ao avaliar extratos metanólicos da casca e do caroço da manga, de diferentes variedades cultivadas no Brasil, observaram riqueza de compostos polifenólicos e destacaram a atividade antioxidante da mangiferina.

O objetivo deste trabalho foi avaliar o efeito da inclusão de extratos etanólicos na ração, obtidos do caroço e da casca da manga, sobre o desempenho de frangos de corte e a oxidação lipídica da carne.

\section{Material e Métodos}

Foram utilizados 360 pintos machos (Gallus gallus domesticus) de um dia de idade, da linhagem Ross 308, distribuídos em delineamento experimental inteiramente casualizado, com seis tratamentos e seis repetições de dez aves.

Os tratamentos consistiram em: ração sem adição de antioxidante (controle); ração com adição de 200 ppm de BHT; ração com 200 ppm de extrato da casca da manga (ECAS); ração com 400 ppm de ECAS; ração com 200 ppm de extrato do caroço da manga (ECAR); e ração com 400 ppm de ECAR.

Foram formuladas rações experimentais isonutrientes para as fases inicial ( 1 a 21 dias de idade), de crescimento (22 a 35 dias de idade) e final (36 a 42 dias de idade) (Tabela 1). Na formulação, foram considerados os valores de composição dos alimentos, conforme Rostagno et al. (2005), e as exigências nutricionais das aves em cada fase, segundo recomendações do manual de manejo da linhagem (Manual de manejo de frangos AgRoss, 2004). O ingrediente inerte da ração foi substituído, de acordo com os tratamentos, pelos antioxidantes.

A preparação dos extratos naturais da manga foi realizada no Laboratório de Produtos Naturais, do Departamento de Química Orgânica e Inorgânica, do Centro de Ciências da Universidade Federal do Ceará (UFC). Cascas e caroços de frutos maduros de mangas das variedades Coité e Jasmim, adquiridos em indústria de processamento de polpas de frutas, foram submetidos à secagem em estufa, a $50^{\circ} \mathrm{C}$, por um período de 24 horas, para as cascas, e de 48 horas para os caroços. Em seguida, o material foi triturado e submetido à extração exaustiva a frio (Barreto et al., 2008). Para isso, os materiais foram colocados em recipientes de vidro, tendo-se adicionado etanol em quantidade suficiente para mantê-los imersos durante sete dias à temperatura ambiente $\left(25^{\circ} \mathrm{C}\right)$. Após a extração, o material foi filtrado, e o extrato foi concentrado em rotaevaporador Büchi Water Bath B-480, (Büchi Labortechnik AG, Flawil, Suíça) a $50^{\circ} \mathrm{C}$, rotação de $60 \mathrm{rpm}$ e pressão reduzida para obtenção do extrato etanólico e do solvente recuperado, o qual foi utilizado para re-extração por mais duas vezes, tendose obedecido às condições da extração inicial.

Como os diferentes extratos etanólicos da manga apresentavam, após a pesagem, características de gel, os extratos foram diluídos em óleo de soja degomado para serem misturados em cada ração.

No início do período experimental, procedeu-se à pesagem das aves de cada parcela. Durante todo o experimento, a ração e a água foram fornecidas à vontade. 
Aágua foi oferecida em bebedouros pendulares e a ração em comedouros tubulares. Ao final de cada fase (inicial, crescimento e final), as sobras de ração e os animais

Tabela 1. Composição $\left(\mathrm{g} \mathrm{kg}^{-1}\right)$ das rações controle para frangos de corte, de acordo com a idade.

\begin{tabular}{lccc}
\hline Ingredientes & \multicolumn{3}{c}{ Idade (dias) } \\
\cline { 2 - 4 } & $1 \mathrm{a} 21$ & $22 \mathrm{a} 35$ & 36 a 42 \\
\hline Milho & 595,6 & 635,8 & 662,8 \\
Farelo de soja & 346,6 & 302,5 & 274,8 \\
Óleo de soja degomado & 18,6 & 25,3 & 27,9 \\
Calcário & 11,1 & 10,0 & 9,9 \\
Fosfato bicálcico & 17,4 & 15,1 & 14,7 \\
DL-metionina & 1,9 & 2,6 & 2,4 \\
Suplemento mineral ${ }^{(1)}$ & 1,0 & 1,0 & 1,0 \\
Suplemento vitamínico & $(2)$ & 3,0 & 2,0 \\
Sal comum & 3,0 & 4,3 & 4,1 \\
Inerte - areia lavada & 4,4 & 0,4 & 0,4 \\
\hline Composição nutricional e energética calculada & & \\
Energia metabolizável $\left(\mathrm{kcal} \mathrm{kg}^{-1}\right)$ & 3.000 & 3.100 & 3.150 \\
Proté́na bruta $\left(\mathrm{g} \mathrm{kg}^{-1}\right)$ & 210,0 & 193,8 & 183,4 \\
Matéria seca $\left(\mathrm{g} \mathrm{kg}^{-1}\right)$ & 864,0 & 866,3 & 866,6 \\
Fibra bruta $\left(\mathrm{g} \mathrm{kg}^{-1}\right)$ & 32,1 & 30,3 & 29,2 \\
Gordura $\left(\mathrm{g} \mathrm{kg}^{-1}\right)$ & 43,8 & 51,2 & 54,4 \\
Cálcio $\left(\mathrm{g} \mathrm{kg}^{-1}\right)$ & 9,0 & 8,0 & 7,8 \\
Fósforo disponível $\left(\mathrm{g} \mathrm{kg}^{-1}\right)$ & 4,5 & 4,0 & 3,9 \\
Fósforo total $\left(\mathrm{g} \mathrm{kg}^{-1}\right)$ & 6,7 & 6,1 & 5,9 \\
Lisina total $\left(\mathrm{g} \mathrm{kg}^{-1}\right)$ & 11,1 & 10,0 & 9,3 \\
Metionina total $\left(\mathrm{g} \mathrm{kg}^{-1}\right)$ & 5,1 & 5,6 & 5,3 \\
Metionina $+\mathrm{cistina}^{-1}$ total $\left(\mathrm{g} \mathrm{kg}^{-1}\right)$ & 8,5 & 8,8 & 8,3 \\
Treonina total $\left(\mathrm{g} \mathrm{kg}^{-1}\right)$ & 8,1 & 7,5 & 7,1 \\
Triptofano total $\left(\mathrm{g} \mathrm{kg}^{-1}\right)$ & 2,6 & 2,4 & 2,2 \\
Potássio $\left(\mathrm{g} \mathrm{kg}^{-1}\right)$ & 8,2 & 7,5 & 7,0 \\
Sódio $\left(\mathrm{g} \mathrm{kg}^{-1}\right)$ & 2,2 & 2,1 & 2,0 \\
Cloro $\left(\mathrm{g} \mathrm{kg}^{-1}\right)$ & 3,0 & 2,9 & 2,8 \\
\hline
\end{tabular}

(1) Suplemento mineral (fornecido por kg do produto): 1 a 21 dias -3.000 $\mathrm{mg}$ de cobre, $12.500 \mathrm{mg}$ de zinco, $12.500 \mathrm{mg}$ de ferro, $16.250 \mathrm{mg}$ de manganês, $250 \mathrm{mg}$ de iodo, $75 \mathrm{mg}$ de selênio, $2,5 \mathrm{~g}$ de antioxidante, $10 \mathrm{~g}$ de coccidiostático, 12,5 g de promotor de crescimento, veículo q.s.p. $1.000 \mathrm{~g} ; 22$ a 35 dias $-3.000 \mathrm{mg}$ de cobre, $12.500 \mathrm{mg}$ de zinco, $12.500 \mathrm{mg}$ de ferro, $16.250 \mathrm{mg}$ de manganês, $250 \mathrm{mg}$ de iodo, $75 \mathrm{mg}$ de selênio, $25 \mathrm{~g}$ de antioxidante, 16,5 g de coccidiostático, 2,5 g de promotor de crescimento, veículo q.s.p. $1.000 \mathrm{~g} ; 35$ a 42 dias $-6.000 \mathrm{mg}$ de cobre, $25.000 \mathrm{mg}$ de zinco, $25.000 \mathrm{mg}$ de ferro, $34.400 \mathrm{mg}$ de manganês, $500 \mathrm{mg}$ de iodo, $45 \mathrm{mg}$ de selênio, 0,625 g de antioxidante, veículo q.s.p. 1.000 g. ${ }^{(2)}$ Suplemento vitamínico (fornecido por $\mathrm{kg}$ do produto): 1 a 21 dias $-1.750 .000 \mathrm{UI}$ de vitamina A, 500.000 UI de vitamina D3, $2.750 \mathrm{mg}$ de vitamina E, $500 \mathrm{mg}$ de vitamina $\mathrm{K} 3,500 \mathrm{mg}$ de vitamina B1, $1.250 \mathrm{mg}$ de vitamina B2, $2.500 \mathrm{mcg}$ de vitamina B12,700 mg de vitamina B6, $200 \mathrm{mg}$ de ácido fólico,

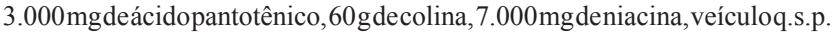
$1.000 \mathrm{~g} ; 22$ a 35 dias - 1.500 .000 UI de vitamina A, 500.000 UI de vitamina D3, $2.500 \mathrm{mg}$ de vitamina E, $500 \mathrm{mg}$ de vitamina $\mathrm{K} 3,350 \mathrm{mg}$ de vitamina $\mathrm{B} 1,1.000 \mathrm{mg}$ de vitamina B2, $2.000 \mathrm{mcg}$ de vitamina B12, $500 \mathrm{mg}$ de vitamina B6, $150 \mathrm{mg}$ de ácido fólico, $2.750 \mathrm{mg}$ de ácido pantotênico, $75 \mathrm{~g}$ de colina, $6.500 \mathrm{mg}$ de niacina, veículo q.s.p. $1.000 \mathrm{~g} ; 35$ a 42 dias -1.250 .000$ UI de vitamina A, 250.000 UI de vitamina D3, $1.250 \mathrm{mg}$ de vitamina E, $940 \mathrm{mg}$ de vitamina K3, $2.475 \mathrm{mg}$ de niacina, veículo q.s.p. $1.000 \mathrm{~g}$. foram pesados, para determinar o consumo de ração (grama por ave), o ganho de peso (grama por ave) e a conversão alimentar. Durante todo o experimento, foi anotada, diariamente, a mortalidade, para correção do consumo de ração e, consequentemente, da conversão alimentar (Sakomura \& Rostagno, 2007).

Para avaliar o efeito dos antioxidantes na estabilidade lipídica da carne, foram analisadas as substâncias reativas ao ácido tiobarbitúrico (TBARS) na carne do peito, no dia do abate (dia zero) e após 15 dias de armazenamento, com refrigeração a $4^{\circ} \mathrm{C}$. Para isso, aos 42 dias de idade, após jejum alimentar de 6 horas, todas as aves de cada parcela foram pesadas; em seguida, foram selecionadas e identificadas quatro aves com pesos próximos ao peso médio da parcela $( \pm 100 \mathrm{~g})$. As aves escolhidas foram encaminhadas ao abatedouro, abatidas por deslocamento cervical, sangradas, escaldadas (água a $60^{\circ} \mathrm{C}$, por $3 \mathrm{~min}$ ), depenadas e evisceradas. Em seguida, procedeu-se à retirada dos peitos inteiros com ossos, os quais foram acondicionados em sacos de plástico identificados por repetição, colocados em isopor com gelo e transportados para o Laboratório de Processamento de Carnes e Pescado, do Departamento de Tecnologia de Alimentos, da UFC. O peito de cada ave foi dividido ao meio e, em seguida, procedeu-se à desossa. Cada metade foi embalada a vácuo, em saco de plástico devidamente identificado. Assim, de cada ave, foram obtidas duas porções de peito, o que totalizou oito porções por repetição. As quatro metades direitas de cada parcela foram utilizadas na determinação do valor de TBARS no dia zero, e as quatro porções esquerdas foram utilizadas para a determinação após 15 dias de refrigeração a $4^{\circ} \mathrm{C}$.

As análises do valor de TBARS, de cada amostra, foram realizadas em duplicata, por meio de técnica descrita por Kang et al. (2001). Em tubo de boca larga, foram pesados aproximadamente $2 \mathrm{~g}$ de carne de frango e adicionados $18 \mathrm{~mL}$ de ácido perclórico $(3,86 \%)$. O conteúdo dos tubos foi homogeneizado em triturador terrutec (Tecnal, Piracicaba, SP), por $15 \mathrm{~s}$, a alta velocidade. O homogeneizado foi filtrado em papel de filtro Whatman ํㅡㄴ 1 . Posteriormente, $2 \mathrm{~mL}$ do filtrado foram colocados em tubo de ensaio, tendose adicionado, em seguida, $2 \mathrm{~mL}$ de solução aquosa $20 \mathrm{mmol} \mathrm{L}^{-1}$ de ácido tiobarbitúrico. Os tubos foram aquecidos em banho-maria fervente por $30 \mathrm{~min}$. A leitura da densidade óptica da solução foi realizada 
em espectrofotômetro, a $531 \mathrm{~nm}$. O valor de TBARS da amostra foi expresso como miligrama (mg) de malonaldeído por quilograma $(\mathrm{kg})$ de carne, e o valor de cada parcela foi considerado a média das quatro amostras analisadas.

As análises estatísticas dos dados foram realizadas com o procedimento GLM (SAS Institute, 2009). As médias foram comparadas pelo teste de Student-Newman-Keuls (SNK), a 5\% de probabilidade. Para os dados de oxidação lipídica, foi adicionado, ao modelo de análise, o efeito do tempo de armazenamento (0 e 15 dias) e da interação tratamento $\mathrm{x}$ tempo de armazenamento.

\section{Resultados e Discussão}

Não foram observadas diferenças significativas entre os tratamentos para todas as variáveis de desempenho avaliadas, o que indica que a adição dos diferentes antioxidantes não influenciou o consumo de ração, o ganho de peso e a conversão alimentar das aves no período avaliado (Tabela 2).

A quantidade de alimento ingerido voluntariamente está relacionada à palatabilidade da ração. Segundo Leeson \& Summers (2001), os ácidos graxos livres gerados durante a hidrólise da gordura podem promover odor e sabor desagradáveis às rações, o que afeta o consumo. Racanicci et al. (2004) relataram que o processo de lipoperoxidação também resulta na formação de substâncias, como aldeídos, cetonas, álcoois, hidrocarbonetos e ácidos, que conferem odor e sabor desagradável ao alimento, o que diminui a palatabilidade da ração e, consequentemente, o consumo pelas aves. No entanto, no presente trabalho, não houve diferença na quantidade de alimento consumido pelas aves, o que indica que a adição dos extratos não alterou a palatabilidade das rações testadas.

$\mathrm{Na}$ avaliação da extensão da oxidação lipídica na carne de peito dos frangos, os níveis de TBARS foram maiores após o armazenamento por 15 dias, em comparação ao dia zero, independentemente do tratamento (Tabela 3). Observou-se que, no dia zero, houve diferença entre os teores de TBARS, com menores valores para a carne dos frangos que receberam BHT e ECAR, na concentração de 400 ppm. Aos 15 dias de armazenamento, apenas a carne de frangos alimentados com rações contendo extratos etanólicos provenientes do caroço de manga (200 e $400 \mathrm{ppm}$ ) diferiram significativamente do tratamento controle, quanto aos valores de TBARS. Com esse período de armazenamento, o extrato ECAR 400 ppm proporcionou valores de TBARS menores até mesmo que o tratamento com o antioxidante sintético. Portanto, entre os componentes avaliados que podem retardar as reações oxidativas que normalmente ocorrem na carne de frangos, os extratos etanólicos do caroço foram os mais eficientes.

A manga é considerada boa fonte de antioxidantes naturais, entre os quais se destaca a mangiferina, um composto fenol glicosilxantona (Puravankara et al.,

Tabela 2. Consumo de ração (kg por ave), ganho de peso ( $k g$ por ave) e conversão alimentar de frangos de corte de 1 a 42 dias de idade, alimentados com rações contendo os antioxidantes butilato de hidroxitolueno (BHT) e extratos da casca (ECAS) e do caroço da manga (ECAR).

\begin{tabular}{|c|c|c|c|c|c|c|c|}
\hline \multirow[t]{2}{*}{ Variáveis } & \multirow[t]{2}{*}{ Controle } & \multirow{2}{*}{$\begin{array}{c}\text { BHT } \\
200 \mathrm{ppm}\end{array}$} & \multicolumn{2}{|c|}{ ECAS } & \multicolumn{2}{|c|}{ ECAR } & \multirow{2}{*}{$\begin{array}{l}\text { CV } \\
(\%)\end{array}$} \\
\hline & & & $200 \mathrm{ppm}$ & $400 \mathrm{ppm}$ & $200 \mathrm{ppm}$ & $400 \mathrm{ppm}$ & \\
\hline Consumo de ração & 4,52 & 4,46 & 4,35 & 4,38 & 4,35 & 4,35 & 2,45 \\
\hline Ganho de peso & 2,52 & 2,50 & 2,46 & 2,41 & 2,43 & 2,45 & 2,83 \\
\hline Conversão alimentar & 1,80 & 1,78 & 1,77 & 1,82 & 1,79 & 1,78 & 2,40 \\
\hline
\end{tabular}

Tabela 3. Estabilidade lipídica da carne quanto às substâncias reativas ao ácido tiobarbitúrico $\left(\mathrm{mg} \mathrm{kg}^{-1}\right)$ em carne de peitos de frangos alimentados com rações contendo os antioxidantes butilato de hidroxitolueno (BHT) e extratos da casca (ECAS) e do caroço da manga (ECAR), em função do tempo de armazenamento $^{(1)}$.

\begin{tabular}{|c|c|c|c|c|c|c|c|}
\hline \multirow[t]{2}{*}{ Armazenamento } & \multirow[t]{2}{*}{ Controle } & \multirow{2}{*}{$\begin{array}{c}\text { BHT } \\
200 \mathrm{ppm}\end{array}$} & \multicolumn{2}{|c|}{ ECAS } & \multicolumn{2}{|c|}{ ECAR } & \multirow{2}{*}{$\begin{array}{l}\text { CV } \\
(\%)\end{array}$} \\
\hline & & & $200 \mathrm{ppm}$ & $400 \mathrm{ppm}$ & $200 \mathrm{ppm}$ & $400 \mathrm{ppm}$ & \\
\hline Dia 0 & $0,19 \mathrm{Ab}$ & $0,16 \mathrm{Bb}$ & $0,18 \mathrm{Ab}$ & $0,19 \mathrm{Ab}$ & $0,19 \mathrm{Ab}$ & $0,14 \mathrm{Bb}$ & 10,44 \\
\hline Dia 15 & $0,57 \mathrm{Aa}$ & $0,52 \mathrm{ABa}$ & $0,56 \mathrm{Aa}$ & $0,52 \mathrm{ABa}$ & $0,46 \mathrm{BCa}$ & $0,43 \mathrm{Ca}$ & 10,44 \\
\hline
\end{tabular}

${ }^{(1)}$ Médias seguidas de letras iguais, maiúsculas nas linhas e minúsculas nas colunas, não diferem, entre si, pelo teste SNK, a 5\% de probabilidade. 
2000; Abdalla et al., 2007). A mangiferina tem atividade antioxidante comprovada (Barreto et al., 2008), e a proporção deste composto nos frutos é variável em função de cultivares e das partes analisadas (Bernardini et al., 2005; Barreto et al., 2008). Schieber et al. (2003) e Barreto et al. (2008) demonstraram que existe diferença entre casca e caroço da manga, quanto ao tipo e ao conteúdo de compostos fenólicos presentes. Os dados apresentados por Ribeiro et al. (2008) corroboram resultados anteriores e indicam que a concentração de compostos fenólicos presentes no caroço da manga da variedade Ubá foi superior em comparação à casca (8,25 vs. $5,72 \%)$. Além disso, de acordo com Abdalla et al. (2007), o caroço da manga é uma excelente fonte de tocoferóis, que também são compostos que apresentam propriedades antioxidantes, potencial fator de inibição de catalisadores oxidativos, como a tirosinase, e atividade antioxidante (Maisuthisakul \& Gordon, 2009). Essas informações estão de acordo com o observado neste trabalho, pois embora os extratos etanólicos do caroço da manga adicionados à ração tenham resultado em menor valor de TBARS na carne de frango, aqueles extraídos da casca da manga não apresentaram o mesmo efeito.

Pereira et al. (2010) verificaram que o extrato etanólico da casca da manga adicionado a mortadelas apresentou efeito antioxidante comparável ao do antioxidante sintético BHT. Já Pereira et al. (2011) relataram ação antioxidante semelhante à do BHT com a adição de 0,1 e $0,2 \%$ de extrato do caroço da manga em mortadelas. Segundo estes autores, a ação antioxidante dos extratos etanólicos da manga pode ser atribuída a substâncias como polifenóis, antocianinas, carotenoides e tocoferóis, presentes nos extratos.

Resultados semelhantes foram obtidos em outros trabalhos sobre o uso de produtos vegetais que contêm compostos com atividade antioxidante, na alimentação de frangos de corte. Florou-Paneri et al. (2005) observaram que a adição de orégano na forma de erva ou de óleo essencial de orégano, em rações para perus, foi capaz de aumentar a estabilidade lipídica da carne fresca do peito e das pernas, uma vez que as amostras da carne das aves alimentadas com essas rações apresentaram menores valores de TBARS. Resultados similares foram relatados por Marcinčák et al. (2008), com a adição de óleo essencial de orégano na ração de frangos, e por Luna et al. (2010), com a adição de 150 ppm de timol e carvacrol à ração.

\section{Conclusões}

1. A adição de extratos etanólicos da manga não afeta os parâmetros de desempenho de frangos de corte de 1 a 42 dias de idade.

2. O extrato etanólico do caroço da manga (ECAR), na dosagem de 200 e 400 ppm, retarda a oxidação lipídica da carne de frangos e, entre os tratamentos avaliados, o ECAR a 400 ppm é o mais eficiente.

\section{Referências}

ABDALLA, A.E.M.; DARWISH, S.M.; AYAD, E.H.E.; EL-HAMAHMY, R.M. Egyptian mango by-product 2: antioxidant and antimicrobial activities of extract and oil from mango seed kernel. Food Chemistry, v.103, p.1141-1152, 2007.

AJILA, C.M.; NAIDU, K.A.; BHAT, S.G.; PRASADA RAO, U.J.S. Bioactive compounds and antioxidant potential of mango peel extract. Food Chemistry, v.105, p.982-988, 2007.

BARRETO, J.C.; TREVISAN, M.T.S.; HULL, W.E.; ERBEN, G.; BRITO, E.S. de; PFUNDSTEIN, B.; WURTELE, G.; SPIEGELHALDER, B.; OWEN, R.W. Characterization and quantitation of polyphenolic compounds in bark, kernel, leaves, and peel of mango (Mangifera indica L.). Journal of Agricultural and Food Chemistry, v.56, p.5599-5610, 2008.

BERNARDINI, N.; FEZER, R.; CONRAD, J.; BEIFUSS, U.; CARLE, R.; SCHIEBER, A. Screening of mango (Mangifera indica $\mathrm{L}$.) cultivars for their contents of flavonol $\mathrm{O}$ - and xanthone C-glycosides, anthocyanins, and pectin. Journal of Agricultural and Food Chemistry, v.53, p.1563-1570, 2005.

BOU, R.; GUARDIOLA, F.; GRAU, A.; GRIMPA, S.; MANICH, A.; BARROETA, A.; CODONY, R. Influence of dietary fat source, $\alpha$-tocopherol, and ascorbic acid supplementation on sensory quality of dark chicken meat. Poltry Science, v.80, p.800-807, 2001.

CHEN, C.H.; PEARSON, A.M.; GRAY, J.I. Effects of synthetic antioxidants (BHA, BHT and PG) on the mutagenicity of IQ-like compounds. Food Chemistry, v.43, p.177-183, 1992.

DURÁN, R.M.; PADILLA, R.B. Actividad antioxidante de los compuestos fenólicos. Grasas e Aceites, v.44, p.101-106, 1993.

FISCHER, G.; BERMUDEZ, V.L.; SIQUEIRA, E.B.; DEL PINO, F.A.B.; ANCIUTI, M.A.; MAIER, J.C.; RUTZ, F. Peroxidação em amostras de milho, protegidas ou não por etoxiquim. Ciência Animal Brasileira, v.6, p.227-232, 2005.

FLOROU-PANERI, P.; PALATOS, G.; GOVARIS, A.; BOTSOGLOU, D.; GIANNENAS, I.; AMBROSIADIS, I. Oregano herbs versus oregano essential oil as feed supplements to increase the oxidative stability of turkey meat. International Journal of Poultry Science, v.4, p.866-871, 2005.

GRAY, J.I.; GOMAA, E.A.; BUCKLEY, D.J. Oxidative quality and shelf life of meats. Meat Science, v.43, p.111-123, 1996.

GROBAS, S.; MÉNDEZ, J.; LOPEZ BOTE, C.; DE BLAS, C.; MATEOS, G.C. Effect of vitamin E and A supplementation on egg 
yolk $\alpha$-tocopherol concentration. Poultry Science, v.81, p.376-381, 2002.

KANG, K.R.; CHERIAN, G.; SIM, J.S. Dietary palm oil alters the lipid stability of polyunsaturated fatty acid-modified poultry products. Poultry Science, v.80, p.228-234, 2001.

LEESON, S.; SUMMERS, D.J. Nutrition of the chicken. $4^{\text {th }}$ ed. Ontario: University Books, 2001. 413p.

LUNA, A.; LÁBAQUE, M.C.; ZYGADLO, J.A.; MARIN, R.H. Effects of thymol and carvacrol feed supplementation on lipid oxidation in broiler meat. Poultry Science, v.89, p.366-370, 2010.

MAISUTHISAKUL, P.; GORDON, M.H. Antioxidant and tyrosinase inhibitory activity of mango seed kernel by-product. Food Chemistry, v.117, p.332-341, 2009.

MANUAL de manejo de frangos AgRoss. Campinas: Agroceres Ross, 2004. 111p.

MARCINČÁK, S.; CABADAJ, R.; POPELKA, P.; SOLTYSOVA, L. Antioxidative effect of oregano supplemented to broilers on oxidative stability of poultry meat. Slovenia Veterinary Research, v.45, p.61-66, 2008.

MARIUTTI, L.R.B.; BRAGAGNOLO, N. A oxidação lipídica em carne de frango e o impacto da adição de sálvia (Salvia officinalis, L.) e de alho (Allium sativum, L.) como antioxidantes naturais. Revista Instituto Adolfo Lutz, v.68, p.1-11, 2009.

MELO, E. de A.; MANCINI FILHO, J.; GUERRA, N.B.; MACIEL, G.R. Atividade antioxidante de extratos de coentro (Coriandrum sativum L.). Ciência e Tecnologia de Alimentos, v.23, p.195-199, 2003.

PEREIRA, A.L.F.; VIDAL, T.F.; TEIXEIRA, M.C.; OLIVEIRA, P. de F.; POMPEU, R.C.F.F.; VIEIRA, M.M.M.; ZAPATA, J.F.F. Antioxidant effect of mango seed extract and butylated hydroxytoluene in bologna-type mortadella during storage. Ciência e Tecnologia de Alimentos, v.31, p.135-140, 2011.
PEREIRA, A.L.F.; VIDAL, T.F.; TEIXEIRA, M.C.; OLIVEIRA, P. de F.; VIEIRA, M.M.M.; ZAPATA, J.F.F.; POMPEU, R.C.F.F.; FREITAS, E.R. Estabilidade oxidativa de mortadelas contendo extrato da casca da manga (Mangifera indica L.). Brazilian Journal of Food Technology, v.13, p.293-298, 2010.

PURAVANKARA, D.; BOHGRA, V.; SHARMA, R.S. Effect of antioxidant principles isolated from mango (Mangifera indica $\mathrm{L}$.) seed kernels on oxidative stability of buffalo ghee (butter-fat). Journal of the Science of Food and Agriculture, v.80, p.522-526, 2000.

RACANICCI, A.M.C.; DANIELSEN, B.; MENTEN, J.F.M.; REGITANO-D'ARCE, M.A.B.; SKIBSTED, L.H. Antioxidant effect of dittany (Origanum dictamnus) in pre-cooked chicken meat balls during chill-storage in comparison to rosemary (Rosmarinus officinalis). European Food Research and Technology, v.218, p.521-524, 2004.

RIBEIRO, S.M.R.; BARBOSA, L.C.A.; QUEIROZ, J.H.; KNODLER, M.; SCHIEBER, A. Phenolic compounds and antioxidant capacity of Brazilian mango (Mangifera indica L.) varieties. Food Chemistry, v.110, p.620-626, 2008.

ROSTAGNO, H.S.; ALBINO, L.F.T.; DONZELE, J.L.; GOMES, P.C.; OLIVEIRA, R.F. de; LOPES, D.C.; FERREIRA, A.S.; BARRETO, S.L.T. Tabelas brasileiras para aves e suínos: composição de alimentos e exigências nutricionais. 2.ed. Viçosa: UFV:DZO, 2005. 186p.

SAKOMURA, N.K.; ROSTAGNO, H.S. Métodos de pesquisa em nutrição de monogástricos. Jaboticabal: Funep, 2007. 283p.

SAS INSTITUTE. SAS/STAT: user's Guide. Version 9.2. Cary: SAS Institute, 2009. 7869p.

SCHIEBER, A.; BERARDINI, N.; CARLE, R. Identification of flavonol and xanthone glycosides from mango (Mangifera indica L. Cv. "Tommy Atkins") peels by high-performance liquid chromatography-electrospray ionization mass spectrometry. Journal of Agricultural and Food Chemistry, v.51, p.5006-5011, 2003.

$\overline{\text { Recebido em } 13 \text { de março de } 2012 \text { e aprovado em } 16 \text { de julho de } 2012}$ 